\title{
Effect of dietary quercetin and sphingomyelin on intestinal nutrient absorption and animal growth
}

\author{
J. Barrenetxe ${ }^{1}$, P. Aranguren ${ }^{1}$, A. Grijalba ${ }^{2}$, J.M. Martínez-Peñuela ${ }^{3}$, F. Marzo ${ }^{1}$ and E. Urdaneta ${ }^{1}{ }^{*}$ \\ ${ }^{1}$ Laboratory of Animal Physiology and Nutrition, School of Agronomy, Public University of Navarra, 31006 Pamplona (Navarra), Spain \\ ${ }^{2}$ Biochemistry Unit, Navarra Hospital, 31008 Pamplona (Navarra), Spain \\ ${ }^{3}$ Anatomo-Pathology Unit, Navarra Hospital, 31008 Pamplona (Navarra), Spain
}

(Received 1 June 2005 - Revised 9 September 2005 - Accepted 18 October 2005)

\begin{abstract}
Research on cancer and other conditions has shown flavonoids and sphingolipids to be food components capable of exerting chemoprotective action. Nevertheless, little is known about their effects on healthy individuals and their potential usefulness as therapeutic agents. The present study examined the possible action of a dietary flavonoid, quercetin, and a sphingolipid, sphingomyelin, as functional foods in healthy animals. In particular, the effect on animal growth of supplementing a conventional diet with one or other of these substances ( $0.5 \%$ quercetin and $0.05 \%$ sphingomyelin) was considered. Possible action affecting intestinal physiology was also analysed by measuring the uptake of sugar and dipeptide, mediated by the Na ${ }^{+}$-dependent sugar transporter SGLT1 and the dipeptide $\mathrm{Na}^{+} / \mathrm{H}^{+}$exchanger PEPT1 respectively, and the activity of related intestinal enzymes such as sucrase, maltase and aminopeptidase $\mathrm{N}$. Both substances seemed to modify small intestinal activity in healthy mice, altering intestinal enzymatic activity and nutrient uptake. These effects observed in the small intestine did not impair normal development of the animals, as no differences in serum biochemical parameters or in organ and body weights were found. The findings should help in elucidating the mechanisms of action of these food components with a view to their possible use in the prevention of certain pathological conditions.
\end{abstract}

Quercetin: Sphingomyelin: SGLT1: PEPT1

As the relationship between health and diet becomes clearer and our understanding of nutrition changes, we are becoming more and more interested in knowing about what we are eating. In recent years attention has focused increasingly on the roles of functional foods in preventing various chronic conditions. Consequently, flavonoids, sphingolipids, fibre and oligosaccharides, food components derived from certain ingredients in fruits, vegetables and milk, have lately come to be widely studied; and their roles in preventing and ameliorating various chronic diseases (cardiovascular disease, allergies, cancer) have been described (Liu, 2004; Halliwell et al. 2005), opening up a new research field in nutrition.

Dietary flavonoids are known to help protect against several pathological conditions, owing mainly to their antioxidant activity, and their possible role as chemopreventive factors has been described (Murota \& Terao, 2003). Quercetin is the most abundant flavonoid in the human diet (Boyer et al. 2004). It is present as a glycoside in a variety of vegetables but reaches the intestinal tract as an aglycone subsequent to the action of the hydrolytic microflora (Gee et al. 2000; Nemeth et al. 2003). Recent investigation has shown quercetin glycosides to exert an influence on in vitro intestinal sugar absorption (Ader et al. 2001; Cermak et al. 2004).
Sphingolipids are structural and functional bioactive lipids found in eggs, milk, meat, fish and soyabeans (Vesper et al. 1999) that regulate cell growth, differentiation and death, and therefore have been looked upon as chemopreventive agents (Lemonnier et al. 2003). Their activity as anticancer factors has been described widely in the literature (Schmelz et al. 2000). In the gastrointestinal tract sphingolipids are hydrolysed to ceramides and sphingoid bases that are absorbed rapidly by the intestinal lining, where they can interact with several intracellular signalling pathways (Schmelz et al. 2001; O'Prey et al. 2003; Moskaug et al. 2004).

Little is known about the effects of these substances as food supplements in the daily diets of healthy individuals. The mechanism of action and the bioavailability of each individual compound must first be understood before there can be any understanding of its bioavailability as a component in the complex overall mixture of foodstuffs ingested. Accordingly, the objective of the present study was to elucidate whether supplementation of the conventional diet by either of these two bioactive factors (the flavonoid quercetin and the sphingolipid sphingomyelin) affects normal animal development and in particular activity by the intestinal tract (absorption and digestion). The results of this research should contribute to our knowledge

Abbreviations: BBMV, brush-border-membrane vesicles; C, control diet/group; PEPT1, dipeptide $\mathrm{Na}^{+} / \mathrm{H}^{+}$exchanger; Q, quercetin-supplemented diet/group; SGLT1, $\mathrm{Na}^{+}$-dependent sugar transporter; SM, sphingomyelin-supplemented diet/group.

* Corresponding author: Dr E. Urdaneta, fax +34948 168930, email elena.urdaneta@unavarra.es 
of these bioactive food components and our understanding of how they act in the organism as a whole.

\section{Materials and methods}

Animals, diets and study design

Swiss mice, $28 \mathrm{~d}$ old and weighing about $20 \mathrm{~g}$, were randomly assigned to one of three dietary groups (twenty to thirty animals per group). The mice were obtained from the colony of Charles River Laboratory Animals in Barcelona, Spain. The mice were housed in cages (five animals per cage) kept in a well-ventilated, thermostatically controlled room $\left(22 \pm 2^{\circ} \mathrm{C}\right)$ with a photoperiod of $12 \mathrm{~h}$ light and $12 \mathrm{~h}$ darkness. One group (group C) was fed a control commercial diet that met all minimum requirements for the proper development of growing animals (AIN-93), while the other two groups were fed the same control diet supplemented with either $0.5 \%$ quercetin (group Q) or $0.05 \%$ sphingomyelin (group SM). Fresh diets were prepared weekly (according to Table 1) with purified ingredients. All diets are isonitrogenous and isoenergetic, and were kept refrigerated at $4^{\circ} \mathrm{C}$. Food and water were available ad libitum to the mice. The body weight of all animals was recorded weekly. At the end of the $28 \mathrm{~d}$ experimental period, the mice were anaesthetized and sacrificed by decapitation. Trunk blood was collected for determinations of serum biochemical parameters, and various organs were extracted and weighed. The jejunum was carefully removed, flushed out with ice-cold saline, frozen in liquid $\mathrm{N}_{2}$ and stored at $-80^{\circ} \mathrm{C}$ until use in preparing the brush-border-membrane vesicles (BBMV) for enzymatic assays and measurement of the intestinal absorption of D-galactose and glycylsarcosine. Before being frozen, a portion of the small intestine and a portion of the colon ( $1 \mathrm{~cm}$ lengths) were taken for histological examination.

The Committee on Animal Care at the Public University of Navarra reviewed and approved the animal care protocol and the killing method to ensure compliance with the guidelines of the Canadian Council on Animal Care (1993).

Table 1. Composition of the three experimental diets

\begin{tabular}{|c|c|c|c|}
\hline & \multicolumn{3}{|c|}{ Diet } \\
\hline & $\mathrm{C}$ & Q & SM \\
\hline \multicolumn{4}{|l|}{ Ingredient (g/kg) } \\
\hline Sphingomyelin & 0.0 & 0.0 & 0.5 \\
\hline Quercetin & 0.0 & $5 \cdot 0$ & 0.0 \\
\hline Lactalbumin* & 137.5 & 137.5 & $137 \cdot 5$ \\
\hline Sucrose & $231 \cdot 2$ & $231 \cdot 2$ & $231 \cdot 2$ \\
\hline Corn starch & $454 \cdot 3$ & $454 \cdot 3$ & $454 \cdot 3$ \\
\hline Olive oil & $80 \cdot 0$ & $80 \cdot 0$ & $80 \cdot 0$ \\
\hline Mineral mix $†$ & $35 \cdot 0$ & $35 \cdot 0$ & $35 \cdot 0$ \\
\hline Vitamin mix $†$ & $10 \cdot 0$ & $10 \cdot 0$ & $10 \cdot 0$ \\
\hline Cellulose & $50 \cdot 0$ & $50 \cdot 0$ & $50 \cdot 0$ \\
\hline Cholineł & $2 \cdot 0$ & $2 \cdot 0$ & $2 \cdot 0$ \\
\hline \multicolumn{4}{|l|}{ Calculated composition } \\
\hline Total protein $(\mathrm{g} / \mathrm{kg})$ & $108 \cdot 0$ & $108 \cdot 0$ & $108 \cdot 0$ \\
\hline Crude energy (MJ/kg) & $17 \cdot 0$ & $17 \cdot 0$ & $17 \cdot 0$ \\
\hline Metabolizable energy (MJ/kg) & $16 \cdot 0$ & $16 \cdot 0$ & $16 \cdot 0$ \\
\hline
\end{tabular}

C, control commercial diet; $\mathrm{Q}$, control diet supplemented with quercetin; SM, control diet supplemented with sphingomyelin

* Lactalbumin: $80 \%$ of pure protein, $4 \%$ lactose (Sigma, St. Louis, MO, USA).

† AIN-93 (Dyets Inc., Bethlehem, PA, USA).

$\$ 99 \%$ in the bitartrate form (Sigma).

\section{Histological analyses}

Tissue samples collected for histological study were immediately fixed in $4 \%$ formalin for $24 \mathrm{~h}$. They were then dehydrated, embedded in paraffin, sliced into $5 \mu \mathrm{m}$ sections and processed by haematoxylin-eosin staining.

\section{Preparation of mouse intestinal brush-border-membrane vesicles}

Crude BBMV were obtained according to the method described by Shirazi-Beechey et al. (1990). The BBMV were prepared from a piece of small intestine taken from every animal in each diet group. The mucosa was re-suspended in a buffer consisting of 100 mM-mannitol and 2 mM-HEPES adjusted to $\mathrm{pH} 7 \cdot 1$ with Tris. The suspension was then homogenized using a Potter-Elvehjem homogenizer (Braun, Melsungen, Germany) at $3000 \mathrm{rpm}$ at $4^{\circ} \mathrm{C}$ for $1 \mathrm{~min}$. Next, $\mathrm{MgCl}_{2}$ was added to a final concentration of $10 \mathrm{mM}$, and the mixture was incubated on ice with continuous icecold shaking for $20 \mathrm{~min}$. The mixture was then centrifuged at $2000 \mathrm{~g}$ for $15 \mathrm{~min}$, and the supernatant was collected and centrifuged at $27000 \mathrm{~g}$ for $30 \mathrm{~min}$. Following centrifugation the supernatant was discarded and the pellet re-suspended in a buffer consisting of $100 \mathrm{~mm}$-mannitol, $0 \cdot 1 \mathrm{mM}^{-\mathrm{MgSO}_{4}}$ and $2 \mathrm{mM}$-HEPES adjusted to $\mathrm{pH} 7.4$ with Tris. After a second precipitation with $\mathrm{MgCl}_{2}$, the mixture was finally centrifuged at $27000 \mathrm{~g}$ for $30 \mathrm{~min}$. The pellet was then re-suspended in a buffer consisting of $300 \mathrm{mM}-$ mannitol, $0 \cdot 1 \mathrm{mM}^{-\mathrm{MgSO}_{4}}$ and $10 \mathrm{~mm}$-HEPES adjusted to $\mathrm{pH} 7 \cdot 4$ with Tris. The BBMV of ten mice were pooled, assayed for protein, diluted to $10 \mathrm{mg}$ BBMV protein/ml, aliquoted and frozen in liquid $\mathrm{N}_{2}$. The final BBMV preparation consisted of right-side microvilli five-fold enriched for sucrase specific activity compared with the initial homogenate.

Protein was assayed using the Bradford diagnostic kit (Bio-Rad Laboratories, Barcelona, Spain) by complexing with Coomassie Blue and reading the change in absorbance at $595 \mathrm{~nm}$ using bovine albumin as standard.

\section{Enzymatic activity}

For each experimental diet group a number of BBMV aliquots were thawed and pooled. Three replications of all enzymatic assays were performed. Sucrase (E.C. $3 \cdot 2 \cdot 1 \cdot 48$ ) and maltase (E.C. 3.2.1.20) activities were determined according to the method described by Dahlqvist (1964) using sucrose and maltose as substrates. After incubation in the presence of the appropriate substrate for $30 \mathrm{~min}$, the glucose released was measured using Kit A-510 from Sigma (St. Louis, MO, USA).

Alkaline phosphatase (E.C. 3.1.3.1) was assayed by measuring the rate of hydrolysis of $p$-nitrophenyl phosphate to $p$-nitrophenol (Uezato \& Fujita, 1983), the results being expressed as U/mg protein.

The activity of a dipeptidase anchored on the brush-border membrane of the enterocytes was also determined (Andria et al. 1980). In the mouse and rat intestine, $\beta$-naphthylamides of L-amino acids are hydrolysed more actively by particle-bound enzymes. Aminopeptidase N (E.C. 3.4.11.2) activity was measured spectrofluorometrically using L-Gly-Pro- $\beta$-naphthylamide as substrate.

Sugar and dipeptide uptake by brush-border-membrane vesicles

Sugar and dipeptide uptake by the BBMV was measured using a slightly modified version of the rapid filtration technique 
developed by Hopfer et al. (1975). Three replications were performed for each experimental diet group. D-Galactose uptake was determined in the presence of an $\mathrm{Na}^{+}$gradient at $37^{\circ} \mathrm{C}$. The incubation medium had a $\mathrm{pH}$ of 7.4 and consisted of $0 \cdot 1 \mathrm{~mm}$-D-galactose, $100 \mathrm{~mm}$-NaSCN, $100 \mathrm{~mm}$-mannitol, $0 \cdot 1 \mathrm{mM}$ $\mathrm{MgSO}_{4}, 10 \mathrm{mM}$-HEPES and the radioactive substrate used as tracer, D- $\left[1-{ }^{14} \mathrm{C}\right]$ galactose $(0 \cdot 037 \mathrm{MBq} / \mathrm{ml}$; Amersham Radiochemical Centre, UK). Glycylsarcosine uptake was measured at $27^{\circ} \mathrm{C}$ in a pH 5.5 medium consisting of $1 \mathrm{~mm}$-glycylsarcosine,

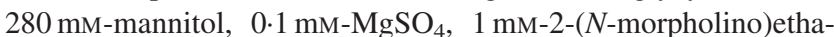
nesulfonic acid and the radioactive substrate used as tracer, $\left[1-{ }^{3} \mathrm{H}\right]$ glycylsarcosine $(0 \cdot 370 \mathrm{MBq} / \mathrm{ml}$; Moravek Biochemicals, Inc., Brea, CA, USA). At the times shown, incubation was halted by adding ice-cold stop solution (150 mM-KSCN, $0.25 \mu \mathrm{M}$-phloridzin and $10 \mathrm{~mm}$-HEPES at $\mathrm{pH} 7.4$ for the galactose uptake determinations and $210 \mathrm{~mm}-\mathrm{KCl}$ and $2 \mathrm{mM}$-HEPES at $\mathrm{pH}$ 7.5 for the dipeptide uptake determinations). The suspension was poured immediately onto a cellulose nitrate filter $(0.45 \mu \mathrm{m}$, $25 \mathrm{~mm}$ diameter; Sartorius, Edgewood, NY, USA) and the filter was then washed twice in ice-cold stop solution and dissolved in HiSafe 3 scintillation liquid for final measurement of radioactivity using a $\beta$-counter.

\section{Western blot analysis}

Expression of transporters PEPT1 $\left(\mathrm{H}^{+} /\right.$peptide cotransporter 1) (Katsura \& Inui, 2003) and SGLT1 $\left(\mathrm{Na}^{+} /\right.$glucose cotransporter 1) in the BBMV from the different experimental diet groups was detected using Western blotting. Samples were diluted in a buffer consisting of $50 \mathrm{~mm}-$ Tris- $\mathrm{HCl}, \mathrm{pH} \mathrm{6.8,2 \%} \mathrm{SDS,} 10 \%$ glycerol, $5 \%$ 2-mercaptoethanol and 0.05 bromophenol blue, and boiled for $3 \mathrm{~min}$. The samples $(50 \mu \mathrm{g})$ were then loaded onto a $12 \% \mathrm{SDS} /$ PAGE mini-gel and separated in an electrophoresis unit for $60 \mathrm{~min}$. The separated proteins were transferred to a nitrocellulose membrane (Hybond P; Amersham-Pharmacia Biotech, Barcelona, Spain) at $250 \mathrm{~mA} / 100 \mathrm{~V}$ and $4^{\circ} \mathrm{C}$ for $2 \mathrm{~h}$. The membranes were blocked with Tris-buffered saline $(100 \mathrm{~mm}$-Tris-HCl, $\mathrm{pH} 7.5$, $0.9 \% \mathrm{NaCl}$ ) containing $0.1 \%$ Tween and $6 \%$ skimmed milk powder at room temperature for $90 \mathrm{~min}$. PEPT1 and SCLT1 were detected by incubating the membranes overnight with respectively a specific affinity-purified rat PEPT1 antiserum kindly donated by Dr Smith (University of Michigan, MI, USA) at a dilution of 1:500 and a rabbit SGLT1 polyclonal antibody (Chemicon Europe Ltd, Hampshire, UK) at a dilution of 1:2500. After being washed three times in Tris-buffered saline containing $0.1 \%$ Tween, the membranes were incubated with the secondary antibody (E0466; Dako Diagnostics, Barcelona, Spain) at a dilution of 1:2500 at room temperature for $1 \mathrm{~h}$. Finally, the immunoreactive proteins were visualized by chemiluminiscence (Pierce Super Signal West Dura; Pierce Biotechnology, Rockford, IL, USA). Bands were quantified by densitometry using the Quantity One program, version 4.5 (Bio-Rad Laboratories, Hertfordshire, UK), and values in the presence of quercetin or sphingomyelin were normalized to those for the control group.

\section{Statistical analysis}

Results given in the text and tables are expressed as means with their standard errors. Statistical analysis of the data was performed by a Kruskal-Wallis one-way ANOVA using SPSS version 11.5 software (SPSS Inc., Chicago, IL, USA). Post hoc

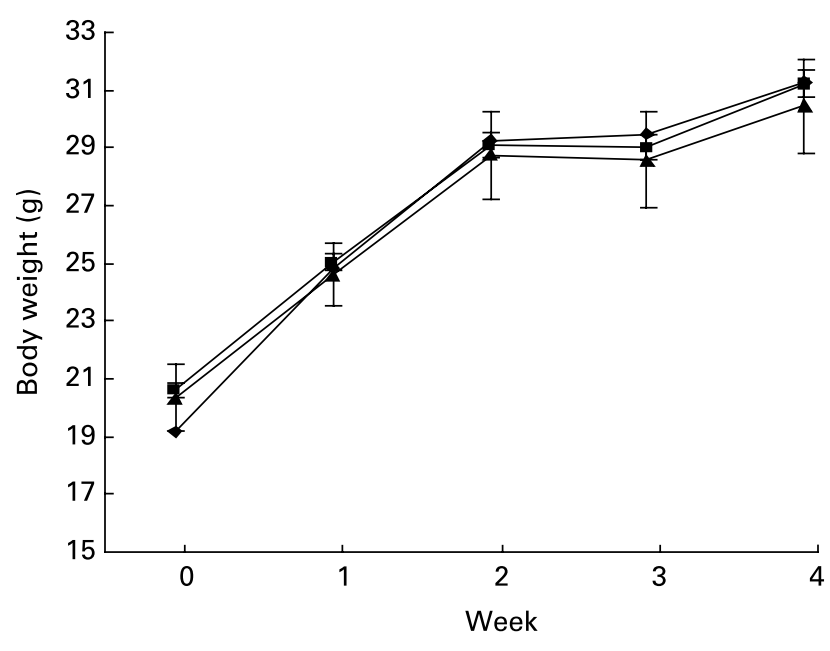

Fig. 1. Body growth of animals fed the different experimental diets for 4 weeks: $(\bullet)$, control commercial diet; $(\square)$, control diet supplemented with $0.5 \%$ quercetin; $(\boldsymbol{\Lambda})$, control diet supplemented with $0.05 \%$ sphingomyelin. Values are means with their standard error shown by vertical bars

comparisons were carried out by a least significant difference procedure. Differences among the groups were deemed statistically significant when $P<0 \cdot 05$.

\section{Results}

\section{Animal growth}

Including quercetin or sphingomyelin in the diet did not alter the final body weight of the animals (Fig. 1), the weight gain (C: 0.43 (SE 0.03), Q: 0.38 (SE 0.02), SM: 0.36 (SE 0.01) g/d) or the body growth index (C: 1.63 (SE 0.08), Q: 1.52 (SE 0.10), SM: 1.50 (SE 0.09)). These findings indicated that neither of the substances tested affected the growth rate of the animals as compared with the control group. Similarly, the serum biochemical parameters measured did not exhibit any differences (Table 2) between the various experimental diet groups other than certain hypoglycaemic and hypocholesterolaemic effects in group SM and a lower triacylglycerol level in group Q.

The weights of the extracted organs (jejunum, pancreas, colon, spleen, and liver) were likewise not altered by quercetin or sphingomyelin intake (data not shown).

It therefore seems that adding these substances to animal diets does not impair the animal's normal growth rates or metabolic status.

\section{Intestinal morphology}

There were no differences in the morphology of the small intestinal epithelium between the three experimental groups (data not shown); hence intake of these food components at the levels tested does not appear to be detrimental to normal development of the small intestine.

Nutrient uptake and enzymatic activity in brush-border-membrane vesicles

The effect of the supplemented diets on the intestinal physiology of the growing mice was also determined. Fig. 2 shows that the activity of the intestinal enzymes assayed was altered when quercetin or sphingomyelin was added to the standard control diet. 
Table 2. Serum biochemical parameters of the animals fed the different experimental diets for 4 weeks (Mean values with their standard errors)

\begin{tabular}{|c|c|c|c|c|c|c|}
\hline \multirow[b]{3}{*}{ Parameter $(n=20)$} & \multicolumn{6}{|c|}{ Diet } \\
\hline & \multicolumn{2}{|c|}{$\mathrm{C}$} & \multicolumn{2}{|c|}{$\mathrm{Q}$} & \multicolumn{2}{|c|}{ SM } \\
\hline & Mean & SE & Mean & SE & Mean & SE \\
\hline Glucose (mg/dl) & $158 \cdot 72$ & 6.83 & $163 \cdot 31$ & 9.96 & $146 \cdot 29$ & $9 \cdot 85$ \\
\hline Urea $(\mathrm{mg} / \mathrm{dl})$ & 57.41 & 2.54 & $50 \cdot 00$ & $2 \cdot 42$ & 51.65 & $3 \cdot 11$ \\
\hline Protein (g/dl) & 3.61 & 0.08 & 3.79 & 0.12 & 3.66 & 0.12 \\
\hline Albumin $(\mathrm{g} / \mathrm{dl})$ & $2 \cdot 81$ & 0.05 & 2.79 & 0.057 & 2.67 & 0.12 \\
\hline Aspartate aminotransferase (U/I) & 55.50 & 3.56 & $48 \cdot 21$ & 4.43 & $50 \cdot 35$ & $5 \cdot 82$ \\
\hline Alanine aminotransferase (U/I) & $15 \cdot 10$ & 1.42 & 13.42 & 0.88 & $14 \cdot 18$ & $1 \cdot 20$ \\
\hline Lactate dehydrogenase (U/I) & $438 \cdot 34$ & 36.62 & $411 \cdot 42$ & $31 \cdot 32$ & $384 \cdot 23$ & 38.26 \\
\hline$\alpha$-Amylase $(U / L)$ & $7120 \cdot 80$ & $208 \cdot 26$ & $7614 \cdot 21$ & $300 \cdot 0$ & 6877.58 & $229 \cdot 30$ \\
\hline Cholesterol (mg/dl) & 105.93 & 3.55 & 108.63 & $4 \cdot 0$ & $100 \cdot 94$ & 4.86 \\
\hline Triacylglycerols (mg/dl) & 71.65 & 5.06 & 64.89 & 3.57 & 73.88 & $5 \cdot 08$ \\
\hline HDL-cholesterol (mg/dl) & $92 \cdot 27$ & 2.96 & 93.47 & 3.24 & 89.94 & 3.91 \\
\hline Cholesterol:HDL-cholesterol & $1 \cdot 14$ & 0.01 & $1 \cdot 16$ & 0.01 & $1 \cdot 12$ & 0.01 \\
\hline
\end{tabular}

In animals fed the diet supplemented with $0.5 \%$ quercetin, there was a slight decrease in intestinal sucrase activity $(P=0.078)$. Maltase activity was distinctly lower $(P<0.001)$ in both group $\mathrm{Q}$ and group $\mathrm{SM}$. In contrast, aminopeptidase $\mathrm{N}$ activity clearly increased $(P<0.001)$ in the animals fed both the experimentally supplemented diets, and alkaline phosphatase activity also increased in group Q $(P<0 \cdot 001)$.

Similarly, the plots of the BBMV uptake assays demonstrated that the presence of quercetin or sphingomyelin in the diet inhibited D-galactose uptake while stimulating glycylsarcosine uptake (Fig. 3). This effect was discernible only when nutrient absorption was mediated by a transporter located in the brushborder membrane (short assay times and an $\mathrm{Na}^{+}$or $\mathrm{H}^{+}$gradient). For longer incubation times ( $10 \mathrm{~min}$ and $60 \mathrm{~min}$, respectively), the sugar and the dipeptide tested entered the vesicles by a process of diffusion that was not altered by the diet.

Looking at all these results together as a whole, it seems that adding quercetin or sphingomyelin to the diet has a differing effect on nutrient absorption and digestion, inhibiting the mechanisms involved in sugar absorption and digestion and enhancing the mechanisms involved in peptide absorption and digestion.

\section{Western blot analysis of SGLT1 and PEPT1 expression}

To elucidate whether these effects on sugar and dipeptide uptake are related to changes in the expression of the transporters involved, the presence of the $\mathrm{Na}^{+}$-dependent sugar transporter SGLT1 and the dipeptide $\mathrm{Na}^{+} / \mathrm{H}^{+}$exchanger PEPT1 on the
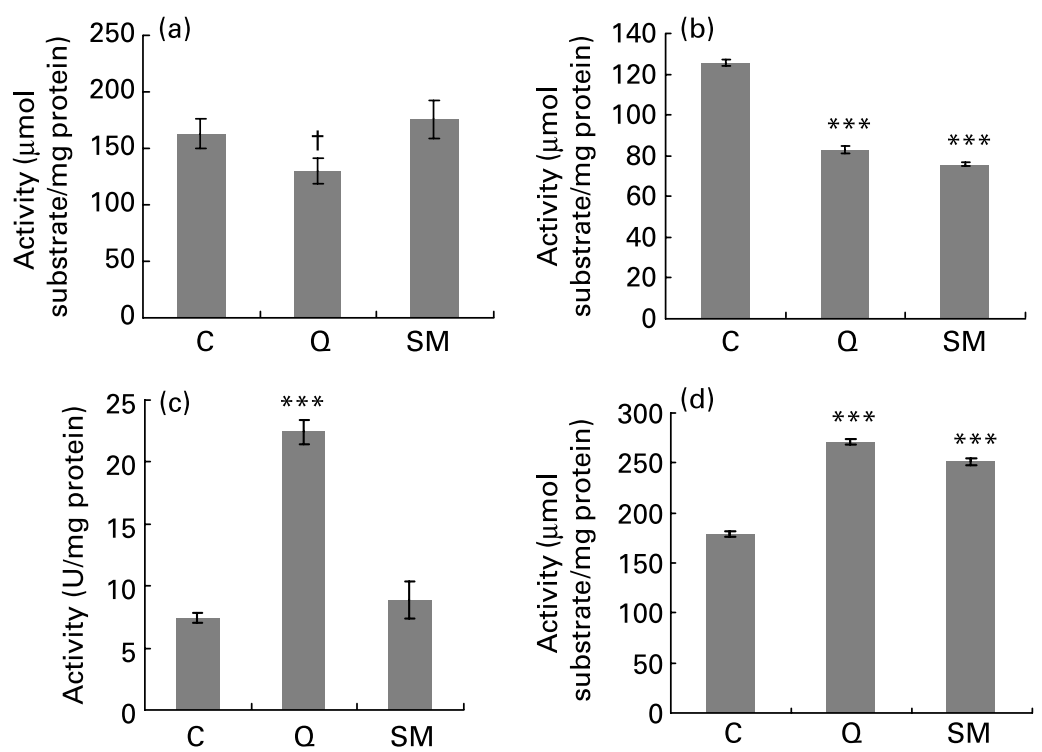

Fig. 2. Sucrase (a), maltase (b), alkaline phosphatase (c) and aminopeptidase $N$ (d) enzymatic activity in intestinal brush-border-membrane vesicles obtained from mice fed the different experimental diets (C, control commercial diet; $Q$, control diet supplemented with $0.5 \%$ quercetin; SM, control diet supplemented with $0.05 \%$ sphingomyelin) for 4 weeks. Values are means with their standard error shown by vertical bars for nine determinations from three different experiments. Mean values were significantly different from those of the control group: $\dagger P=0.078,{ }^{\star \star \star} P<0.001$ 

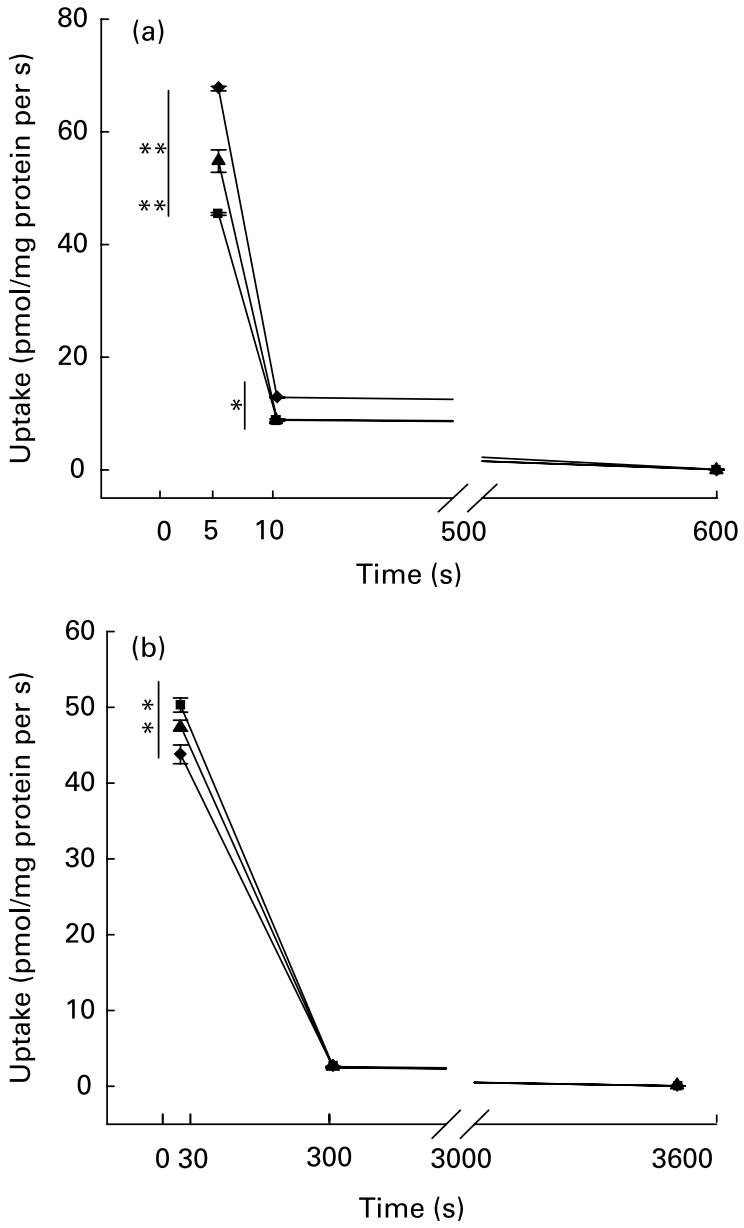

Fig. 3. Uptake of (a) $1 \mathrm{~mm}-\mathrm{D}$-galactose (in the presence of an $\mathrm{Na}^{+}$gradient at $37^{\circ} \mathrm{C}$ and $\mathrm{pH} 7.4$ ) and (b) $0.1 \mathrm{~mm}$-glycylsarcosine (in the presence of an $\mathrm{H}^{+}$gradient at $27^{\circ} \mathrm{C}$ and $\mathrm{pH} 5.5$ ) by intestinal brush-border-membrane vesicles obtained from mice fed the different experimental diets ( $\bullet$, control commercial diet; $\mathbf{\square}$, control diet supplemented with $0.5 \%$ quercetin; $\boldsymbol{\Lambda}$, control diet supplemented with $0.05 \%$ sphingomyelin) for 4 weeks. Values are means with their standard error shown by vertical bars for one representative experiment of three different determinations. Mean values were significantly different from those of the control group: ${ }^{\star} P<0.05$, ${ }^{\star \star} P<0.01$

apical membrane of the enterocytes was analysed by Western blotting.

Fig. 4 depicts a clear increase in expression of the PEPT1 transporter in the BBMV solutions obtained from the animals in group SM and a slight decrease in those from group Q, compared with group C. It thus seems that the effect of higher dipeptide uptake in the two supplemented groups was due to different mechanisms for quercetin and for sphingomyelin.

Expression of SGLT1 in the two supplemented diet groups increased compared with the control group and was highest for group Q. These results suggest that the lower galactose absorption observed in the uptake assays described above could be brought about by a loss of transporter affinity for its substrate.

\section{Discussion}

The roles of quercetin and sphingomyelin in the development of several chronic diseases have been discussed widely in the literature, and it has been suggested that they may possibly act as bioactive factors. However, little is known about the effects of these substances on healthy individuals and their potential for use as preventive or therapeutic agents. This makes it necessary to analyse their effects on healthy organisms in order to be able to formulate reliable recommendations concerning their possible use in preventive therapies and establish a scientifically sound basis for determining their effective doses, possible sideeffects, etc.

The present study analysed the effects of quercetin and sphingomyelin on the growth, development and intestinal physiology of healthy mice, since the intestinal barrier regulates the entry of nutrients and other substances that affect the individual's nutritional status. Maintaining the proper nutritional status is of great importance in the prevention and development of illness.

The doses of quercetin and sphingomyelin tested were selected based on other reports (Lemonnier et al. 2003) and data that have estimated levels of daily intake in man as ranging between 10 and $20 \mathrm{mg}$ for quercetin (De Vries et al. 1997) and 0.3 and $0.5 \mathrm{~g}$ (0.01-0.02\% of the diet by weight) for sphingolipids (Vesper et al. 1999; Merrill et al. 1997) in the populations of Western societies. Accordingly, since the mice had a total food intake of approximately $5 \mathrm{~g} / \mathrm{d}$, supplementation of $0.5 \%$ quercetin in the experimental diet represented a daily intake of $25 \mathrm{mg}$ quercetin, which is in the range of values reported for intake by human subjects. Supplementation of $0.05 \%$ sphingomyelin was likewise equivalent to amounts that have been observed to exert detectable effects in different studies performed on mice (Schmelz et al. 1996) and was close to the estimated intake levels for man mentioned above.

The normal growth rate and metabolic status of the animals were not affected by inclusion of either substance in the diets, suggesting that at the doses tested they could be added as dietary supplements without detrimental effect. This finding is in agreement with other studies on mice performed by other workers, which have shown that administering sphingomyelin in the diet did not alter weight gain by animals (Dillehay et al. 1994; Sang \& Sung, 2003).

In contrast, the determinations of activity by the small intestine revealed that ingesting quercetin or sphingomyelin did affect intestinal enzymatic activity and nutrient absorption, although the morphology of the jejunum was not altered. Unexpectedly, while the activity of enzymes involved in glucose absorption (sucrase and maltase) decreased, the activity of enzymes involved in dipeptide absorption (aminopeptidase $\mathrm{N}$ ) increased. This was consistent with the results of the assays of galactose and glycylsarcosine uptake by the BBMV. The presence of quercetin or sphingomyelin in the diet inhibited D-galactose uptake and stimulated glycylsarcosine uptake. Recently, other researchers observed quercetin to have a similar effect in glucose uptake studies performed using porcine intestinal BBMV (Cermak et al. 2004) and isolated rat adipocytes (Strobel et al. 2005). In addition, regulation by quercetin of intestinal disaccharidases in diabetic rats has also been described (Ramachandra et al. 2005).

These findings could be explained by an action of quercetin or sphingomyelin on transporter expression levels, altering transporter availability on the apical membrane of the enterocytes. To confirm this hypothesis, expression of the $\mathrm{Na}^{+}$-dependent sugar transporter SGLT1 and the dipeptide $\mathrm{Na}^{+} / \mathrm{H}^{+}$exchanger PEPT1 was measured by the Western blot method. These analyses revealed changes in the expression of both PEPT1 and SGLT1 in mice fed the experimental diets supplemented with quercetin 
(a)

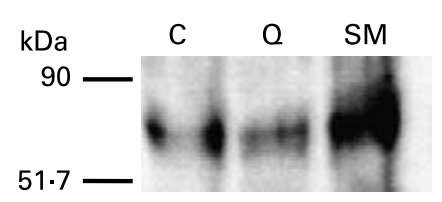

(b)

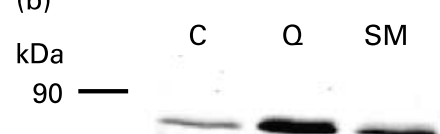

$51 \cdot 7$
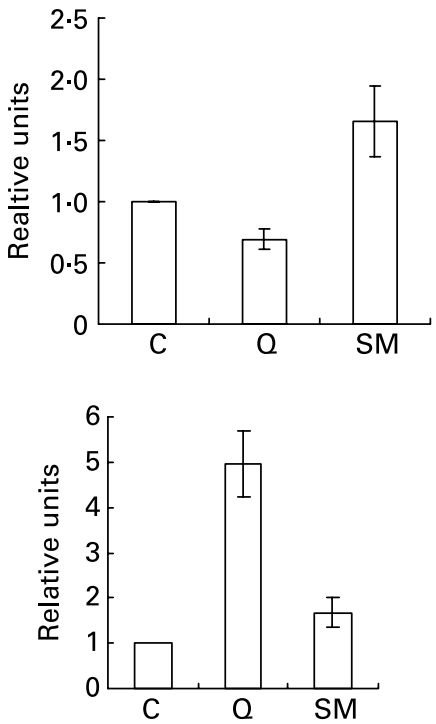

Fig. 4. Representative Western blot (left) and corresponding densitometric analysis (right) showing regulation of (a) the dipeptide $\mathrm{Na}^{+} / \mathrm{H}^{+}$exchanger $\mathrm{PEPT} 1$ and (b) the $\mathrm{Na}^{+}$-dependent sugar transporter SGLT1 in intestinal brush-border-membrane vesicles obtained from mice fed the different experimental diets (C, control commercial diet; Q, control diet supplemented with $0.5 \%$ quercetin; SM, control diet supplemented with $0.05 \%$ sphingomyelin) for 4 weeks. As expected, in both cases a band of approximately $75-80 \mathrm{kDa}$ corresponding to the protein under study was observed. In the densitometric analysis, values are means with their standard error shown by vertical bars for three different blots

and sphingomyelin, expression of SGLT1 being increased and expression of PEPT1 being decreased, although the latter only in the presence of supplementation with quercetin.

It therefore seems that even though similar effects were observed in the BBMV uptake assays, regulation of the dipeptide transporter by these two substances differs. The PEPT1 transporter is known to be regulated by diet (Shiraga et al. 1999; Adibi, 2003), and the higher expression of this transporter in the sphingomyelin-supplemented diet could be due in part to lower levels of di- or tripeptides in the intestinal lumen brought about by the increased aminopeptidase $\mathrm{N}$ activity recorded. It has also been suggested that quercetin and sphingomyelin or their metabolic derivatives enter the enterocytes and might interact with various intracellular signalling pathways (O'Prey et al. 2003), which in turn affects regulation of transporter turnover processes. Stimulation of PEPT1 activity by quercetin by increasing the maximal rate of uptake has already been demonstrated in other studies (Wenzel et al. 2001).

Surprisingly, however, the lower galactose uptake in the BBMV was consistent with higher expression of the SGLT1 transporter, suggesting that quercetin and sphingomyelin might affect membrane insertion of the protein, thereby altering its affinity for its substrate and decreasing its transport activity. In addition, lower sugar levels in the intestinal lumen due to the decreased activity of maltase and sucrase referred to above could also contribute to this effect on galactose absorption.

The regulation of nutrient uptake observed in the small intestine did not impair normal development of the animals, as no differences in serum biochemical parameters or in organ and body weights were found. This fact could be interesting in malnutrition situations established as a result of some pathological diseases such as cancer, cirrhosis and inflammatory bowel disease.

In conclusion, both these bioactive substances seem clearly to modify small intestine activity in healthy mice, altering intestinal enzymatic activity and nutrient uptake. It is therefore of great interest to elucidate all possible beneficial and/or detrimental action that these compounds might have on the organism as a whole before recommending them for use in the therapeutic treatment of different pathological conditions. Further study is needed to identify the mechanisms of action by quercetin and sphingomyelin on intestinal physiology in order to lay out the scientific basis for their use in the prevention of chronic diseases.

\section{Acknowledgements}

We thank Dr Smith from the University of Michigan who kindly donated the PEPT1 antibody used in the present study. This work was supported by a grant from the Health Department of the Navarra Government.

\section{References}

Ader P, Blöck M, Pietzsch S \& Wolffram S (2001) Interaction of quercetin glucosides with the sodium/glucose cotransporter (SGLT1). Cancer Lett 162, 175-180.

Adibi SA (2003) Regulation of the intestinal oligopeptide transporter (Pept-1) in health and disease. Am J Physiol Gastrointest Liver Physiol 285, G779-G788.

Andria G, Cucchiara S, De Vizia B, De Ritis G, Masaka G \& Aurichio S (1980) Brush border and cytosol peptidase activities of human small intestine in normal subjects and celiac patients. Pediatr Res 14, 812-818i.

Boyer J, Brown D \& Liu RH (2004) Uptake of quercetin and quercetin 3 -glucoside from whole onion and apple peel extracts by Caco-2 cell monolayers. J Agric Food Chem 52, 7172-7179.

Cermak R, Landgraf S \& Wolffram S (2004) Quercetin glucosides inhibit glucose uptake into brush-border-membrane vesicles of porcine jejunum. Br J Nutr 91, 849-855.

Dahlqvist A (1964) Method for assay of intestinal disaccharidases. Anal Biochem 7, 18-25 
De Vries JHM, Janssen PL, Hollman PCH, van Staveren WA \& Katan MB (1997) Consumption of quercetin and kaempferol in free-living subjects eating a variety of diets. Cancer Lett 114, 141-144.

Dillehay DL, Webb SK, Schmelz EM \& Merrill AH Jr (1994) Dietary sphingomyelin inhibits 1,2-dimethylhydrazine-induced colon cancer in CF1 mice. $J$ Nutr 124, 615-620.

Gee JM, Du Pont MS, Day AJ, Plumb GW, Williamson G \& Johnson IT (2000) Intestinal transport of quercetin glycosides in rats involves both deglycosylation and interaction with the hexose transport pathway. $J$ Nutr 130, 2765-2771.

Halliwell B, Rafter J \& Jenner J (2005) Health promotion by flavonoids, tocopherols, tocotrienols, and other phenols: direct or indirect effects? Antioxidant or not? Am J Clin Nutr 81, 268-276.

Hopfer U, Sigrist-Nelson K, Perotto J \& Murer H (1975) Intestinal sugar transport: studies with isolated plasma membranes. Ann N Y Acad Sci 264, 414-427.

Katsura T \& Inui K (2003) Intestinal absorption of drugs mediated by drug transporters: mechanisms and regulation. Drug Metab Pharmacokinet 18, $1-15$.

Lemonnier LA, Dillehay DL, Vespremi MJ, Abrams J, Brody E \& Schmelz EM (2003) Sphingomyelin in the suppression of colon tumors: prevention versus intervention. Arch Biochem Biophys 419, 129-138.

Liu RH (2004) Potential synergy of phytochemicals in cancer prevention: mechanism of action. J Nutr 134, 3479-3485.

Merrill AH Jr, Schmelz EM, Wang E, Dillehay DL, Rice LG, Meredith F \& Riley RT (1997) Importance of sphingolipids and inhibitors of sphingolipid metabolism as components of animal diets. J Nutr 127, $830 \mathrm{~S}-833 \mathrm{~S}$

Moskaug JO, Carlsen H, Myhrstad M \& Blomhoff R (2004) Molecular imaging of the biological effects of quercetin and quercetin rich foods. Mech Ageing Dev 125, 315-324.

Murota K \& Terao J (2003) Antioxidative flavonoid quercetin: implication of its intestinal absorption and metabolism. Arch Biochem Biophys 417, $12-17$.

Nemeth K, Plumb GW, Berrin JG, Juge N, Jacob R, Naim HY, Williamson G, Swallow DM, \& Kroon PA (2003) Deglycosylation by small intestinal epithelial cell beta-glucosidases is a critical step in the absorption and metabolism of dietary flaronoid glycosides in humans. Eur J Nutr 42, 29-42.

O'Prey J, Brown J, Fleming J \& Harrison PR (2003) Effects of dietary flavonoids on major signal transduction pathways in human epithelial cells. Biochem Pharmacol 66, 2075-2088.
Ramachandra R, Shetty AK \& Salimath PV (2005) Quercetin alleviates activities of intestinal and renal disaccharidases in streptozotocininduced diabetic rats. Mol Nutr Food Res 49, 355-360.

Sang KN \& Sung IK (2003) Egg sphingomyelin lowers the lymphatic absorption of cholesterol and $\alpha$-tocopherol in rats. $J$ Nutr 133, 3571-3576.

Schmelz EM, Dillehay DL, Webb SK, Reiter A, Adams J \& Merrill AH Jr (1996) Sphingomyelin consumption suppresses aberrant colonic crypt foci and increases the proportion of adenomas versus adenocarcinomas in CF1 mice treated with 1,2-dimethylhydrazine: implications for dietary sphingolipids and colon carcinogenesis. Cancer Res 56, 4936-4941.

Schmelz EM, Sullards MC, Dillehay DL \& Merrill AH Jr (2000) Colonic cell proliferation and aberrant crypt foci formation are inhibited by dairy glycosphingolipids in 1,2-dimethylhydrazine-treated CF1 mice. J Nutr 130, 522-527.

Schmelz EM, Roberts PC, Kustin EM, Lemonnier LA, Sullards MC, Dillehay DL \& Merrill AH Jr (2001) Modulation of intracellular $\beta$ catenin localization and intestinal tumorigenesis in vivo and in vitro by sphingolipids. Cancer Res 61, 6723-6729.

Shiraga T, Miyamoto K, Tanaka H, Yamamoto H, Taketani Y, Morita K, Tamai I, Tsuji A \& Takeda E (1999) Cellular and molecular mechanisms of dietary regulation on rat intestinal $\mathrm{H}^{+}$/peptide transporter PepT1. Gastroenterology 116, 354-362.

Shirazi-Beechey SP, Davies AG, Tebbutt K, Dyer J, Ellis A, Taylor CJ, Fairclough P \& Beechey RB (1990) Preparation and properties of brush border membrane vesicles from human small intestine. Gastroenterology 98, 676-685.

Strobel P, Allard C, Pérez-Acle T, Calderon R, Aldunate R \& Leighton F (2005) Myricetin, quercetin and catechin-gallate inhibit glucose uptake in isolated adipocytes. Biochem $J$ 386, 471-478.

Uezato T \& Fujita M (1983) Developmental transition of alkaline phosphatase from suckling to adult type in rat small intestine: molecular species and effect of injected cortisone and thyroxine. J Biochem 94, 1483-1488.

Vesper H, Schmelz EM, Nikolova-Karakashian MN, Dillehay DL, Lynch DV \& Merrill AH Jr (1999) Sphingolipids in food and the emerging importance of sphingolipids to nutrition. J Nutr 129, 1239-1250.

Wenzel U, Kuntz S \& Daniel H (2001) Flavonoids with epidermal growth factor-receptor tyrosine kinase inhibitory activity stimulates PEPT1mediated cefixime uptake into human epithelial cells. J Pharmacol Exp Ther 299, 351-357. 